\title{
STARLIKE AND CONVEXITY PROPERTIES FOR P-VALENT SOLUTIONS OF THE SHAH DIFFERENTIAL EQUATION
}

\begin{abstract}
M. M. Sheremeta, Yu. S. Trukhan. Starlike and convexity properties for $p$-valent solutions of the Shah differential equation, Mat. Stud. 48 (2017), 14-23.

The starlikeness and the convexity in the unit disc and the growth of an entire function $f(z)=z^{p}+\sum_{n=p+1}^{\infty} f_{n} z^{n}, p \in \mathbb{N}$, satisfying the differential equation $z^{2} w^{\prime \prime}+\left(\beta_{0} z^{2}+\beta_{1} z\right) w^{\prime}+$ $+\left(\gamma_{0} z^{2}+\gamma_{1} z+\gamma_{2}\right) w=0\left(\beta_{0}, \beta_{1}, \gamma_{0}, \gamma_{1}, \gamma_{2}\right.$ are complex parameters $)$ are studied.
\end{abstract}

1. Introduction. An analytic function univalent in $\mathbb{D}=\{z:|z|<1\}$

$$
f(z)=\sum_{k=0}^{\infty} f_{k} z^{k}
$$

is said to be convex if $f(\mathbb{D})$ is a convex domain. It is well known $[1$, p. 203] that the condition $\operatorname{Re}\left\{1+z f^{\prime \prime}(z) / f^{\prime}(z)\right\}>0(z \in \mathbb{D})$ is necessary and sufficient for the convexity of $f$. According to W. Kaplan ([2]), a function $f$ is said to be close-to-convex in $\mathbb{D}$ (see also [1, p. 583]) if there exists a convex in $\mathbb{D}$ function $\Phi$ such that $\operatorname{Re}\left(f^{\prime}(z) / \Phi^{\prime}(z)\right)>0(z \in \mathbb{D})$. Every close-to-convex function in $\mathbb{D}$ is univalent in $\mathbb{D}$ and, therefore, $f^{\prime}(0) \neq 0$. Hence it follows that the function $f$ is close-to-convex in $\mathbb{D}$ if and only if the function $(f(z)-f(0)) / f^{\prime}(0)$ is close-to-convex in $\mathbb{D}$. It is clear that $(f(z)-f(0)) / f^{\prime}(0)=g(z)$, where

$$
g(z)=z+\sum_{k=2}^{\infty} g_{k} z^{k}
$$

and $g_{k}=f_{k} / f_{1}$. The function $g$ is said to be starlike if $\operatorname{Re}\left\{z g^{\prime}(z) / g(z)\right\}>0(z \in \mathbb{D})$.

S.M. Shah [3] indicated conditions on real parameters $\beta_{0}, \beta_{1}, \gamma_{0}, \gamma_{1}, \gamma_{2}$ of the differential equation

$$
z^{2} w^{\prime \prime}+\left(\beta_{0} z^{2}+\beta_{1} z\right) w^{\prime}+\left(\gamma_{0} z^{2}+\gamma_{1} z+\gamma_{2}\right) w=0
$$

under which there exists an entire transcendental solution given by (1) such that $f$ and all its derivatives are close-to-convex in $\mathbb{D}$. In particular he obtained the following result: if $\beta_{1}+\gamma_{2}=0,-1 \leq \beta_{0}<0, \beta_{1}>0, \gamma_{0}=0$ and $-\beta_{1} / 2<\gamma_{1} \leq 0$, then equation (3) has an entire solution (2) such that all $g^{(n)}(n \geq 0)$ are close-to-convex in $\mathbb{D}$ and $\ln M_{g}(r)=(1+o(1))\left|\beta_{0}\right| r$ as $r \rightarrow+\infty$, where $M_{g}(r)=\max \{|g(z)|:|z|=r\}$.

2010 Mathematics Subject Classification: 30B10, 30C45, 35B08.

Keywords: starlikeness; convexity; entire function; differential equation. doi:10.15330/ms.48.1.14-23

(C) M. M. Sheremeta, Yu. S. Trukhan, 2017 
The investigations are continued in papers [4]-[12]. In particular, in the case of complex parameters $\beta_{0}, \beta_{1}, \gamma_{0}, \gamma_{1}, \gamma_{2}$ in [8] it is proved that if $\gamma_{0}=0, \beta_{1}+\gamma_{2}=0, \beta_{0} \neq 0,\left|\beta_{1}\right|<2$ and $\frac{2\left(\left|\beta_{1}\right|+\left|\gamma_{1}\right|\right)}{2-\left|\beta_{1}\right|}<\ln 2$ then equation (3) has an entire solution $(2)$ such that all $g^{(n)}(n \geq 0)$ are starlike and, thus, close-to-convex in $\mathbb{D}$ and $\ln M_{g}(r)=(1+o(1))\left|\beta_{0}\right| r$ as $r \rightarrow+\infty$. An analog of this assertion for convex functions is obtained in [9], where it is proved that if $\gamma_{0}=0, \beta_{1}+\gamma_{2}=0, \beta_{0} \neq 0,\left|\beta_{1}\right|<2$ and $\frac{2\left(\left|\beta_{1}\right|+\left|\gamma_{1}\right|\right)}{2-\left|\beta_{1}\right|}<\frac{\ln 2}{2}$ then equation (3) has an entire solution $(2)$ such that all $g^{(n)}(n \geq 0)$ are convex in $\mathbb{D}$.

Here we study properties of the solution of (3) of the form

$$
f(z)=z^{p}+\sum_{n=p+1}^{\infty} f_{n} z^{n}, \quad p \in \mathbb{N} .
$$

2. Preliminary results. Let $S(p)$ be the class of functions of the form (4), which are analytic and $p$-valent in the unit disk $\mathbb{D}$. A function $f \in S(p)$ is said to be p-valent starlike of order $\alpha \in[0, p)$ if $\operatorname{Re}\left\{z f^{\prime}(z) / f(z)\right\}>\alpha(z \in \mathbb{D})$. Also a function $f \in S(p)$ is said to be $p$-valent convex of order $\alpha \in[0, p)$ if $\operatorname{Re}\left\{1+z f^{\prime \prime}(z) / f^{\prime}(z)\right\}>\alpha(z \in \mathbb{D})$.

We need the following lemmas.

Lemma 1. ([13], [14]) Let $f \in S(p)$ and $\alpha \in[0, p)$. If

$$
\sum_{n=p+1}^{\infty}(n-\alpha)\left|f_{n}\right| \leq p-\alpha
$$

then $f$ is $p$-valent starlike of order $\alpha$, and if

$$
\sum_{n=p+1}^{\infty} n(n-\alpha)\left|f_{n}\right| \leq p(p-\alpha)
$$

then $f$ is $p$-valent convex of order $\alpha$.

Substituting (4) into (3) we have

$$
\begin{aligned}
p(p-1) z^{p}+ & \sum_{n=p+1}^{\infty} n(n-1) f_{n} z^{n}+\beta_{0} p z^{p+1}+\sum_{n=p+2}^{\infty} \beta_{0}(n-1) f_{n-1} z^{n}+ \\
+\beta_{1} p z^{p}+ & \sum_{n=p+1}^{\infty} \beta_{1} n f_{n} z^{n}+\gamma_{0} z^{p+2}+\sum_{n=p+3}^{\infty} \gamma_{0} f_{n-2} z^{n}+\gamma_{1} z^{p+1}+ \\
& +\sum_{n=p+2}^{\infty} \gamma_{1} f_{n-1} z^{n}+\gamma_{2} z^{p}+\sum_{n=p+1}^{\infty} \gamma_{2} f_{n} z^{n} \equiv 0
\end{aligned}
$$

whence we obtain the following lemma.

Lemma 2. The function (4) is a solution of differential equation (3) if and only if

$$
\begin{gathered}
p\left(p+\beta_{1}-1\right)+\gamma_{2}=0 \\
\left((p+1)\left(p+\beta_{1}\right)+\gamma_{2}\right) f_{p+1}+\beta_{0} p+\gamma_{1}=0 \\
\left((p+2)\left(p+1+\beta_{1}\right)+\gamma_{2}\right) f_{p+2}+\left(\beta_{0}(p+1)+\gamma_{1}\right) f_{p+1}+\gamma_{0}=0
\end{gathered}
$$

and for $n \geq p+3$

$$
\left(n\left(n+\beta_{1}-1\right)+\gamma_{2}\right) f_{n}+\left(\beta_{0}(n-1)+\gamma_{1}\right) f_{n-1}+\gamma_{0} f_{n-2}=0 .
$$


3. Main result. Suppose that for all $n \geq p+1$

$$
n\left(n+\beta_{1}-1\right)+\gamma_{2} \neq 0 .
$$

Then (7)-(10) imply

$$
\begin{gathered}
\gamma_{2}=-p\left(p+\beta_{1}-1\right) \\
f_{p+1}=-\frac{\gamma_{1}}{2 p+\beta_{1}}-\frac{\beta_{0} p}{2 p+\beta_{1}} \\
f_{p+2}=-\frac{\beta_{0}(p+1)+\gamma_{1}}{2\left(2 p+1+\beta_{1}\right)} f_{p+1}-\frac{\gamma_{0}}{2\left(2 p+1+\beta_{1}\right)}
\end{gathered}
$$

and for $n \geq p+3$

$$
f_{n}=-\frac{\beta_{0}(n-1)+\gamma_{1}}{(n-p)\left(n+p-1+\beta_{1}\right)} f_{n-1}-\frac{\gamma_{0}}{(n-p)\left(n+p-1+\beta_{1}\right)} f_{n-2} .
$$

Therefore, if $\left|\beta_{1}\right|<2 p$,

$$
\begin{aligned}
& \sum_{n=p+1}^{\infty}(n-\alpha)\left|f_{n}\right| \leq \frac{\left|\gamma_{1}\right|(p+1-\alpha)}{2 p-\left|\beta_{1}\right|}+\frac{\left|\beta_{0}\right| p(p+1-\alpha)}{2 p-\left|\beta_{1}\right|}+ \\
& +(p+2-\alpha)\left\{\frac{\left|\beta_{0}\right|(p+1)+\left|\gamma_{1}\right|}{2\left(2 p+1-\left|\beta_{1}\right|\right)}\left|f_{p+1}\right|+\frac{\left|\gamma_{0}\right|}{2\left(2 p+1-\left|\beta_{1}\right|\right)}\right\}+ \\
& +\sum_{n=p+3}^{\infty} \frac{(n-\alpha)\left(\left|\beta_{0}\right|(n-1)+\left|\gamma_{1}\right|\right)}{(n-p)\left(n+p-1-\left|\beta_{1}\right|\right)}\left|f_{n-1}\right|+\sum_{n=p+3}^{\infty} \frac{(n-\alpha)\left|\gamma_{0}\right|}{(n-p)\left(n+p-1-\left|\beta_{1}\right|\right)}\left|f_{n-2}\right|= \\
& =\frac{\left|\gamma_{1}\right|(p+1-\alpha)}{2 p-\left|\beta_{1}\right|}+\frac{\left|\beta_{0}\right| p(p+1-\alpha)}{2 p-\left|\beta_{1}\right|}+ \\
& +(p+2-\alpha)\left\{\frac{\left|\beta_{0}\right|(p+1)+\left|\gamma_{1}\right|}{2\left(2 p+1-\left|\beta_{1}\right|\right)}\left|f_{p+1}\right|+\frac{\left|\gamma_{0}\right|}{2\left(2 p+1-\left|\beta_{1}\right|\right)}\right\}+ \\
& +\sum_{n=p+2}^{\infty} \frac{(n+1-\alpha)\left(\left|\beta_{0}\right| n+\left|\gamma_{1}\right|\right)}{(n+1-p)\left(n+p-\left|\beta_{1}\right|\right)}\left|f_{n}\right|+\sum_{n=p+1}^{\infty} \frac{(n+2-\alpha)\left|\gamma_{0}\right|}{(n+2-p)\left(n+p+1-\left|\beta_{1}\right|\right)}\left|f_{n}\right|= \\
& =\frac{\left|\gamma_{1}\right|(p+1-\alpha)}{2 p-\left|\beta_{1}\right|}+\frac{\left|\beta_{0}\right| p(p+1-\alpha)}{2 p-\left|\beta_{1}\right|}+ \\
& +(p+2-\alpha)\left\{\frac{\left|\beta_{0}\right|(p+1)+\left|\gamma_{1}\right|}{2\left(2 p+1-\left|\beta_{1}\right|\right)}\left|f_{p+1}\right|+\frac{\left|\gamma_{0}\right|}{2\left(2 p+1-\left|\beta_{1}\right|\right)}\right\}- \\
& -\frac{(p+2-\alpha)\left(\left|\beta_{0}\right|(p+1)+\left|\gamma_{1}\right|\right)}{2\left(2 p+1-\left|\beta_{1}\right|\right)}\left|f_{p+1}\right|+\sum_{n=p+1}^{\infty} \frac{(n+1-\alpha)\left(\left|\beta_{0}\right| n+\left|\gamma_{1}\right|\right)}{(n-\alpha)(n+1-p)\left(n+p-\left|\beta_{1}\right|\right)}(n-\alpha)\left|f_{n}\right|+ \\
& +\sum_{n=p+1}^{\infty} \frac{(n+2-\alpha)\left|\gamma_{0}\right|}{(n-\alpha)(n+2-p)\left(n+p+1-\left|\beta_{1}\right|\right)}(n-\alpha)\left|f_{n}\right|
\end{aligned}
$$

and, thus,

$$
\sum_{n=p+1}^{\infty}\left\{1-\frac{(n+1-\alpha)\left(\left|\beta_{0}\right| n+\left|\gamma_{1}\right|\right)}{(n-\alpha)(n+1-p)\left(n+p-\left|\beta_{1}\right|\right)}-\frac{(n+2-\alpha)\left|\gamma_{0}\right| /(n-\alpha)}{(n+2-p)\left(n+p+1-\left|\beta_{1}\right|\right)}\right\}(n-\alpha)\left|f_{n}\right| \leq
$$




$$
\leq \frac{\left|\gamma_{1}\right|(p+1-\alpha)}{2 p-\left|\beta_{1}\right|}+\frac{\left|\beta_{0}\right| p(p+1-\alpha)}{2 p-\left|\beta_{1}\right|}+\frac{(p+2-\alpha)\left|\gamma_{0}\right|}{2\left(2 p+1-\left|\beta_{1}\right|\right)}
$$

It is easy to verify that the sequences

$$
\left(\frac{(n+1-\alpha)\left(\left|\beta_{0}\right| n+\left|\gamma_{1}\right|\right)}{(n-\alpha)(n+1-p)\left(n+p-\left|\beta_{1}\right|\right)}\right)_{n \geq p+1}, \quad\left(\frac{(n+2-\alpha)\left|\gamma_{0}\right|}{(n-\alpha)(n+2-p)\left(n+p+1-\left|\beta_{1}\right|\right)}\right)_{n \geq p+1}
$$

are decreasing. Therefore,

$$
\begin{gathered}
\left\{1-\frac{(p+2-\alpha)\left(\left|\beta_{0}\right|(p+1)+\left|\gamma_{1}\right|\right)}{2(p+1-\alpha)\left(2 p+1-\left|\beta_{1}\right|\right)}-\frac{(p+3-\alpha)\left|\gamma_{0}\right|}{3(p+1-\alpha)\left(2 p+2-\left|\beta_{1}\right|\right)}\right\} \sum_{n=p+1}^{\infty}(n-\alpha)\left|f_{n}\right| \leq \\
\leq \frac{\left|\gamma_{1}\right|(p+1-\alpha)}{2 p-\left|\beta_{1}\right|}+\frac{\left|\beta_{0}\right| p(p+1-\alpha)}{2 p-\left|\beta_{1}\right|}+\frac{(p+2-\alpha)\left|\gamma_{0}\right|}{2\left(2 p+1-\left|\beta_{1}\right|\right)}
\end{gathered}
$$

and if

$$
\frac{(p+2-\alpha)\left(\left|\beta_{0}\right|(p+1)+\left|\gamma_{1}\right|\right)}{2(p+1-\alpha)\left(2 p+1-\left|\beta_{1}\right|\right)}+\frac{(p+3-\alpha)\left|\gamma_{0}\right|}{3(p+1-\alpha)\left(2 p+2-\left|\beta_{1}\right|\right)}<1
$$

then

$$
\sum_{n=p+1}^{\infty}(n-\alpha)\left|f_{n}\right| \leq \frac{\frac{\left|\gamma_{1}\right|(p+1-\alpha)}{2 p-\left|\beta_{1}\right|}+\frac{(p+2-\alpha)\left|\gamma_{0}\right|}{2\left(2 p+1-\left|\beta_{1}\right|\right)}+\frac{\left|\beta_{0}\right| p(p+1-\alpha)}{2 p-\left|\beta_{1}\right|}}{1-\frac{(p+2-\alpha)\left(\left|\beta_{0}\right|(p+1)+\left|\gamma_{1}\right|\right)}{2(p+1-\alpha)\left(2 p+1-\left|\beta_{1}\right|\right)}-\frac{(p+3-\alpha)\left|\gamma_{0}\right|}{3(p+1-\alpha)\left(2 p+2-\left|\beta_{1}\right|\right)}}
$$

that is the condition (5) holds if

$$
\frac{\frac{\left|\gamma_{1}\right|(p+1-\alpha)}{2 p-\left|\beta_{1}\right|}+\frac{(p+2-\alpha)\left|\gamma_{0}\right|}{2\left(2 p+1-\left|\beta_{1}\right|\right)}+\frac{\left|\beta_{0}\right| p(p+1-\alpha)}{2 p-\left|\beta_{1}\right|}}{1-\frac{(p+2-\alpha)\left(\left|\beta_{0}\right|(p+1)+\left|\gamma_{1}\right|\right)}{2(p+1-\alpha)\left(2 p+1-\left|\beta_{1}\right|\right)}-\frac{(p+3-\alpha)\left|\gamma_{0}\right|}{3(p+1-\alpha)\left(2 p+2-\left|\beta_{1}\right|\right)}} \leq p-\alpha .
$$

On the other hand, if $\left|\beta_{1}\right|<2 p$,

$$
\begin{gathered}
\sum_{n=p+1}^{\infty} n(n-\alpha)\left|f_{n}\right| \leq \frac{\left|\gamma_{1}\right|(p+1)(p+1-\alpha)}{2 p-\left|\beta_{1}\right|}+\frac{\left|\beta_{0}\right| p(p+1)(p+1-\alpha)}{2 p-\left|\beta_{1}\right|}+ \\
+(p+2)(p+2-\alpha)\left\{\frac{\left|\beta_{0}\right|(p+1)+\left|\gamma_{1}\right|}{2\left(2 p+1-\left|\beta_{1}\right|\right)}\left|f_{p+1}\right|+\frac{\left|\gamma_{0}\right|}{2\left(2 p+1-\left|\beta_{1}\right|\right)}\right\}+ \\
+\sum_{n=p+3}^{\infty} \frac{n(n-\alpha)\left(\left|\beta_{0}\right|(n-1)+\left|\gamma_{1}\right|\right)}{(n-p)\left(n+p-1-\left|\beta_{1}\right|\right)}\left|f_{n-1}\right|+\sum_{n=p+3}^{\infty} \frac{n(n-\alpha)\left|\gamma_{0}\right|}{(n-p)\left(n+p-1-\left|\beta_{1}\right|\right)}\left|f_{n-2}\right|= \\
=\frac{\left|\gamma_{1}\right|(p+1)(p+1-\alpha)}{2 p-\left|\beta_{1}\right|}+\frac{\left|\beta_{0}\right| p(p+1)(p+1-\alpha)}{2 p-\left|\beta_{1}\right|}+ \\
+(p+2)(p+2-\alpha)\left\{\frac{\left|\beta_{0}\right|(p+1)+\left|\gamma_{1}\right|}{2\left(2 p+1-\left|\beta_{1}\right|\right)}\left|f_{p+1}\right|+\frac{\left|\gamma_{0}\right|}{2\left(2 p+1-\left|\beta_{1}\right|\right)}\right\}+ \\
+\sum_{n=p+2}^{\infty} \frac{(n+1)(n+1-\alpha)\left(\left|\beta_{0}\right| n+\left|\gamma_{1}\right|\right)}{(n+1-p)\left(n+p-\left|\beta_{1}\right|\right)}+\sum_{n=p+1}^{\infty} \frac{(n+2)(n+2-\alpha)\left|\gamma_{0}\right|}{(n+2-p)\left(n+p+1-\left|\beta_{1}\right|\right)}\left|f_{n}\right|= \\
=\frac{\left|\gamma_{1}\right|(p+1)(p+1-\alpha)}{2 p-\left|\beta_{1}\right|}+\frac{\left|\beta_{0}\right| p(p+1)(p+1-\alpha)}{2 p-\left|\beta_{1}\right|}+
\end{gathered}
$$




$$
\begin{gathered}
+(p+2)(p+2-\alpha)\left\{\frac{\left|\beta_{0}\right|(p+1)+\left|\gamma_{1}\right|}{2\left(2 p+1-\left|\beta_{1}\right|\right)}\left|f_{p+1}\right|+\frac{\left|\gamma_{0}\right|}{2\left(2 p+1-\left|\beta_{1}\right|\right)}\right\}+ \\
+\sum_{n=p+1}^{\infty} \frac{(n+1)(n+1-\alpha)\left(\left|\beta_{0}\right| n+\left|\gamma_{1}\right|\right)}{n(n-\alpha)(n+1-p)\left(n+p-\left|\beta_{1}\right|\right)} n(n-\alpha)\left|f_{n}\right|+ \\
+\sum_{n=p+1}^{\infty} \frac{(n+2)(n+2-\alpha)\left|\gamma_{0}\right|}{n(n-\alpha)(n+2-p)\left(n+p+1-\left|\beta_{1}\right|\right)} n(n-\alpha)\left|f_{n}\right|- \\
-\frac{(p+2)(p+2-\alpha)\left(\left|\beta_{0}\right|(p+1)+\left|\gamma_{1}\right|\right)}{2\left(2 p+1-\left|\beta_{1}\right|\right)}\left|f_{p+1}\right|,
\end{gathered}
$$

and, thus,

$$
\begin{gathered}
\sum_{n=p+1}^{\infty}\left\{1-\frac{(n+1)(n+1-\alpha)\left(\left|\beta_{0}\right| n+\left|\gamma_{1}\right|\right)}{n(n-\alpha)(n+1-p)\left(n+p-\left|\beta_{1}\right|\right)}-\right. \\
\left.-\frac{(n+2)(n+2-\alpha)\left|\gamma_{0}\right|}{n(n-\alpha)(n+2-p)\left(n+p+1-\left|\beta_{1}\right|\right)}\right\} n(n-\alpha)\left|f_{n}\right| \leq \\
\leq \frac{\left|\gamma_{1}\right|(p+1)(p+1-\alpha)}{2 p-\left|\beta_{1}\right|}+\frac{\left|\beta_{0}\right| p(p+1)(p+1-\alpha)}{2 p-\left|\beta_{1}\right|}+\frac{(p+2)(p+2-\alpha)\left|\gamma_{0}\right|}{2\left(2 p+1-\left|\beta_{1}\right|\right)} .
\end{gathered}
$$

It is easy to verify that the sequences

$$
\left(\frac{(n+1)(n+1-\alpha)\left(\left|\beta_{0}\right| n+\left|\gamma_{1}\right|\right)}{n(n-\alpha)(n+1-p)\left(n+p-\left|\beta_{1}\right|\right)}\right)_{n \geq p+1},\left(\frac{(n+2)(n+2-\alpha)\left|\gamma_{0}\right|}{n(n-\alpha)(n+2-p)\left(n+p+1-\left|\beta_{1}\right|\right)}\right)_{n \geq p+1}
$$

are decreasing. Therefore,

$$
\begin{aligned}
& \left\{1-\frac{(p+2)(p+2-\alpha)\left(\left|\beta_{0}\right|(p+1)+\left|\gamma_{1}\right|\right)}{2(p+1)(p+1-\alpha)\left(2 p+1-\left|\beta_{1}\right|\right)}-\frac{(p+3)(p+3-\alpha)\left|\gamma_{0}\right|}{3(p+1)(p+1-\alpha)\left(2 p+2-\left|\beta_{1}\right|\right)}\right\} \times \\
& \quad \times \sum_{n=p+1}^{\infty} n(n-\alpha)\left|f_{n}\right| \leq \frac{(p+1)(p+1-\alpha)\left(\left|\gamma_{1}\right|+\left|\beta_{0}\right| p\right)}{2 p-\left|\beta_{1}\right|}+\frac{(p+2)(p+2-\alpha)\left|\gamma_{0}\right|}{2\left(2 p+1-\left|\beta_{1}\right|\right)}
\end{aligned}
$$

and if

$$
\frac{(p+2)(p+2-\alpha)\left(\left|\beta_{0}\right|(p+1)+\left|\gamma_{1}\right|\right)}{2(p+1)(p+1-\alpha)\left(2 p+1-\left|\beta_{1}\right|\right)}+\frac{(p+3)(p+3-\alpha)\left|\gamma_{0}\right|}{3(p+1)(p+1-\alpha)\left(2 p+2-\left|\beta_{1}\right|\right)}<1
$$

then

$$
\sum_{n=p+1}^{\infty} n(n-\alpha)\left|f_{n}\right| \leq \frac{\frac{(p+1)(p+1-\alpha)\left(\left|\gamma_{1}\right|+\left|\beta_{0}\right| p\right)}{2 p-\left|\beta_{1}\right|}+\frac{(p+2)(p+2-\alpha)\left|\gamma_{0}\right|}{2\left(2 p+1-\left|\beta_{1}\right|\right)}}{1-\frac{(p+2)(p+2-\alpha)\left(\left|\beta_{0}\right|(p+1)+\left|\gamma_{1}\right|\right)}{2(p+1)(p+1-\alpha)\left(2 p+1-\left|\beta_{1}\right|\right)}-\frac{(p+3)(p+3-\alpha)\left|\gamma_{0}\right|}{3(p+1)(p+1-\alpha)\left(2 p+2-\left|\beta_{1}\right|\right)}},
$$

that is the condition (6) holds if

$$
\frac{\frac{(p+1)(p+1-\alpha)\left(\left|\gamma_{1}\right|+\left|\beta_{0}\right| p\right)}{2 p-\left|\beta_{1}\right|}+\frac{(p+2)(p+2-\alpha)\left|\gamma_{0}\right|}{2\left(2 p+1-\left|\beta_{1}\right|\right)}}{1-\frac{(p+2)(p+2-\alpha)\left(\left|\beta_{0}\right|(p+1)+\left|\gamma_{1}\right|\right)}{2(p+1)(p+1-\alpha)\left(2 p+1-\left|\beta_{1}\right|\right)}-\frac{(p+3)(p+3-\alpha)\left|\gamma_{0}\right|}{3(p+1)(p+1-\alpha)\left(2 p+2-\left|\beta_{1}\right|\right)}} \leq p(p-\alpha)
$$


Theorem 1. Let $\alpha \in[0, p), p\left(p+\beta_{1}-1\right)+\gamma_{2}=0,\left|\beta_{1}\right|<2 p$ and (11) holds for all $n \geq p+1$. Then differential equation (3) has an entire solution (4), which under the condition

$$
\begin{gathered}
\frac{(p+1-\alpha)\left(\left|\gamma_{1}\right|+\left|\beta_{0}\right| p\right)}{(p-\alpha)\left(2 p-\left|\beta_{1}\right|\right)}+\frac{(p+2-\alpha)\left|\gamma_{0}\right|}{2(p-\alpha)\left(2 p+1-\left|\beta_{1}\right|\right)}+ \\
+\frac{(p+2-\alpha)\left(\left|\beta_{0}\right|(p+1)+\left|\gamma_{1}\right|\right)}{2(p+1-\alpha)\left(2 p+1-\left|\beta_{1}\right|\right)}+\frac{(p+3-\alpha)\left|\gamma_{0}\right|}{3(p+1-\alpha)\left(2 p+2-\left|\beta_{1}\right|\right)} \leq 1
\end{gathered}
$$

is $p$-valent starlike of order $\alpha$ in $\mathbb{D}$ and under the condition

$$
\begin{gathered}
\frac{\left(\left|\gamma_{1}\right|+\left|\beta_{0}\right| p\right)(p+1)(p+1-\alpha)}{p(p-\alpha)\left(2 p-\left|\beta_{1}\right|\right)}+\frac{(p+2)(p+2-\alpha)\left|\gamma_{0}\right|}{2 p(p-\alpha)\left(2 p+1-\left|\beta_{1}\right|\right)}+ \\
+\frac{(p+2)(p+2-\alpha)\left(\left|\beta_{0}\right|(p+1)+\left|\gamma_{1}\right|\right)}{2(p+1)(p+1-\alpha)\left(2 p+1-\left|\beta_{1}\right|\right)}+\frac{(p+3)(p+3-\alpha)\left|\gamma_{0}\right|}{3(p+1)(p+1-\alpha)\left(2 p+2-\left|\beta_{1}\right|\right)} \leq 1
\end{gathered}
$$

is $p$-valent convex of order $\alpha$ in $\mathbb{D}$, and $\ln M_{f}(r)=(1+o(1)) \sigma r$ as $r \rightarrow+\infty$, where $M_{f}(r)=$ $=\max \{|f(z)|:|z|=r\}$ and either $\sigma=\frac{1}{2}\left|-\beta_{0}+\sqrt{\beta_{0}^{2}-4 \gamma_{0}}\right|$ or $\sigma=\frac{1}{2}\left|-\beta_{0}-\sqrt{\beta_{0}^{2}-4 \gamma_{0}}\right|$.

Proof. The condition (20) implies (16) and (17). Therefore, (5) holds and by Lemma 1 the function (4) is $p$-valent starlike of order $\alpha$. On the other hand, the condition (21) implies (18) and (19). Therefore, (6) holds and, by Lemma 1, the function (4) is $p$-valent convex of order $\alpha$. The asymptotic equality $\ln M_{f}(r)=(1+o(1)) \sigma r$ as $r \rightarrow+\infty$ is proved in [8] using Wiman-Valiron's method.

4. Appendix. Suppose that (11) does not hold, that is, for example $(p+1)\left(p+\beta_{1}\right)+\gamma_{2}=0$. Then in view of (7) we have $\beta_{1}=-2 p$, and (8) implies the equality $\gamma_{1}+\beta_{0} p=0$. Hence it follows that $f_{p+1}$ can be arbitrary. We choose $f_{p+1}=0$. Then from (14) and (15) we obtain $f_{p+2}=-\frac{\gamma_{0}}{2}$ and $f_{n}=-\frac{\beta_{0}}{n-p} f_{n-1}-\frac{\gamma_{0}}{(n-p)(n-p-1)} f_{n-2}$ for $n \geq p+3$. Therefore, as above,

$$
\begin{aligned}
& \sum_{n=p+1}^{\infty}(n-\alpha)\left|f_{n}\right|=\sum_{n=p+2}^{\infty}(n-\alpha)\left|f_{n}\right| \leq \frac{\left|\gamma_{0}\right|(p+2-\alpha)}{2}+\sum_{n=p+3}^{\infty} \frac{(n-\alpha)\left|\beta_{0}\right|}{n-p}\left|f_{n-1}\right|+ \\
& \quad+\sum_{n=p+3}^{\infty} \frac{(n-\alpha)\left|\gamma_{0}\right|}{(n-p)(n-p-1)}\left|f_{n-2}\right|=\frac{\left|\gamma_{0}\right|(p+2-\alpha)}{2}+ \\
& +\sum_{n=p+2}^{\infty} \frac{(n+1-\alpha)\left|\beta_{0}\right|}{n+1-p}\left|f_{n}\right|+\sum_{n=p+2}^{\infty} \frac{(n+2-\alpha)\left|\gamma_{0}\right|}{(n+2-p)(n-p+1)}\left|f_{n}\right|=\frac{\left|\gamma_{0}\right|(p+2-\alpha)}{2}+ \\
& +\sum_{n=p+2}^{\infty}\left(\frac{(n+1-\alpha)\left|\beta_{0}\right|}{(n-\alpha)(n+1-p)}+\frac{(n+2-\alpha)\left|\gamma_{0}\right|}{(n-\alpha)(n+2-p)(n-p+1)}\right)(n-\alpha)\left|f_{n}\right|,
\end{aligned}
$$

whence

$$
\sum_{n=p+2}^{\infty}\left(1-\frac{(p+3-\alpha)\left|\beta_{0}\right|}{3(p+2-\alpha)}-\frac{(p+4-\alpha)\left|\gamma_{0}\right|}{12(p+2-\alpha)}\right)(n-\alpha)\left|f_{n}\right| \leq \frac{\left|\gamma_{0}\right|(p+2-\alpha)}{2}
$$

If

$$
\frac{4(p+3-\alpha)\left|\beta_{0}\right|+(p+4-\alpha)\left|\gamma_{0}\right|}{12(p+2-\alpha)}<1
$$


then

$$
\sum_{n=p+1}^{\infty}(n-\alpha)\left|f_{n}\right| \leq \frac{6(p+2-\alpha)^{2}\left|\gamma_{0}\right|}{12(p+2-\alpha)-4(p+3-\alpha)\left|\beta_{0}\right|-(p+4-\alpha)\left|\gamma_{0}\right|}
$$

Similarly,

$$
\begin{gathered}
\sum_{n=p+1}^{\infty} n(n-\alpha)\left|f_{n}\right|=\sum_{n=p+2}^{\infty} n(n-\alpha)\left|f_{n}\right| \leq \frac{\left|\gamma_{0}\right|(p+2)(p+2-\alpha)}{2}+ \\
+\sum_{n=p+3}^{\infty} \frac{n(n-\alpha)\left|\beta_{0}\right|}{n-p}\left|f_{n-1}\right|+\sum_{n=p+3}^{\infty} \frac{n(n-\alpha)\left|\gamma_{0}\right|}{(n-p)(n-p-1)}\left|f_{n-2}\right|=\frac{\left|\gamma_{0}\right|(p+2)(p+2-\alpha)}{2}+ \\
+\sum_{n=p+2}^{\infty}\left(\frac{(n+1)(n+1-\alpha)\left|\beta_{0}\right|}{n(n-\alpha)(n+1-p)}+\frac{(n+2)(n+2-\alpha)\left|\gamma_{0}\right|}{n(n-\alpha)(n+2-p)(n-p+1)}\right) n(n-\alpha)\left|f_{n}\right|
\end{gathered}
$$

and if

$$
\frac{(p+3)(p+3-\alpha)\left|\beta_{0}\right|}{3(p+2)(p+2-\alpha)}+\frac{(p+4)(p+4-\alpha)\left|\gamma_{0}\right|}{12(p+2)(p+2-\alpha)}<1
$$

then

$$
\begin{aligned}
& \sum_{n=p+1}^{\infty} n(n-\alpha)\left|f_{n}\right| \leq \\
& \leq \frac{6(p+2)^{2}(p+2-\alpha)^{2}\left|\gamma_{0}\right|}{12(p+2)(p+2-\alpha)-4(p+3)(p+3-\alpha)\left|\beta_{0}\right|-(p+4)(p+4-\alpha)\left|\gamma_{0}\right|} .
\end{aligned}
$$

Therefore, as above we obtain the following statement.

Proposition 1. Let $\alpha \in[0, p), \gamma_{1}+\beta_{0} p=0, \beta_{1}=-2 p$ and $\gamma_{2}=p(p+1)$. Then differential equation (3) has an entire solution

$$
f(z)=z^{p}-\frac{\gamma_{0} z^{p+2}}{2}+\sum_{n=p+3}^{\infty} f_{n} z^{n}
$$

where $f_{n}=-\frac{\beta_{0}}{n-p} f_{n-1}-\frac{\gamma_{0}}{(n-p)(n-p-1)} f_{n-2}$ for $n \geq p+3$, which under the condition

$$
\frac{\left|\gamma_{0}\right|(p+2-\alpha)}{2(p-\alpha)}+\frac{4(p+3-\alpha)\left|\beta_{0}\right|+(p+4-\alpha)\left|\gamma_{0}\right|}{12(p+2-\alpha)} \leq 1
$$

is $p$-valent starlike of order $\alpha$ in $\mathbb{D}$ and under the condition

$$
\frac{\left|\gamma_{0}\right|(p+2)(p+2-\alpha)}{2 p(p-\alpha)}+\frac{(p+3)(p+3-\alpha)\left|\beta_{0}\right|}{3(p+2)(p+2-\alpha)}+\frac{(p+4)(p+4-\alpha)\left|\gamma_{0}\right|}{12(p+2)(p+2-\alpha)} \leq 1
$$

is $p$-valent convex of order $\alpha$ in $\mathbb{D}$.

Indeed, for the function (26) the condition (27) implies (22) and in view of (23) we have (5). In view of (25) the condition (28) implies (24) and we obtain (6). Therefore, by Lemma 1 Proposition 1 is proved. 
5. Polynomial solutions. Suppose that a function

$$
f(z)=z^{p}+\sum_{n=p+1}^{p+k} f_{n} z^{n}, \quad f_{p+k} \neq 0
$$

satisfies the differential equation (3), that is $f_{n}=0$ for $n \geq p+k+1$. Then from (15) we obtain

$$
\frac{\beta_{0}(p+k)+\gamma_{1}}{(k+1)\left(2 p+k+\beta_{1}\right)} f_{p+k}-\frac{\gamma_{0}}{(k+1)\left(2 p+k+\beta_{1}\right)} f_{p+k-1}=-f_{p+k+1}=0
$$

and

$$
\frac{\beta_{0}(p+k+1)+\gamma_{1}}{(k+2)\left(2 p+k+1+\beta_{1}\right)} f_{p+k+1}+\frac{\gamma_{0}}{(k+2)\left(2 p+k+1+\beta_{1}\right)} f_{p+k}=-f_{p+k+2}=0 .
$$

Hence it follows that $\gamma_{0}=0$ and $\beta_{0}(p+k)+\gamma_{1}=0$ and, thus, if $k \geq 3$ then for $p+3 \leq n \leq p+k$ $f_{n}=\frac{(p+k+1-n) \beta_{0}}{(n-p)\left(n+p-1+\beta_{1}\right)} f_{n-1}$. Also (14) and (13) imply $f_{p+2}=\frac{\beta_{0}(k-1)}{2\left(2 p+1+\beta_{1}\right)} f_{p+1}$ and $f_{p+1}=\frac{\beta_{0} k}{2 p+\beta_{1}}$. Therefore, if $\left|\beta_{1}\right|<2 p$,

$$
\begin{gathered}
\sum_{n=p+1}^{p+k}(n-\alpha)\left|f_{n}\right| \leq \frac{\left|\beta_{0}\right| k(p+1-\alpha)}{2 p-\left|\beta_{1}\right|}+\frac{\left|\beta_{0}\right|(k-1)(p+2-\alpha)}{2\left(2 p+1-\left|\beta_{1}\right|\right)}\left|f_{p+1}\right|+ \\
+\sum_{n=p+3}^{p+k} \frac{(n-\alpha)(p+k+1-n)\left|\beta_{0}\right|}{(n-p)\left(n+p-1-\left|\beta_{1}\right|\right)}\left|f_{n-1}\right|= \\
=\frac{\left|\beta_{0}\right| k(p+1-\alpha)}{2 p-\left|\beta_{1}\right|}+\frac{\left|\beta_{0}\right|(k-1)(p+2-\alpha)}{2\left(2 p+1-\left|\beta_{1}\right|\right)}\left|f_{p+1}\right|+ \\
+\sum_{n=p+2}^{p+k-1} \frac{(n+1-\alpha)(p+k-n)\left|\beta_{0}\right|}{(n+1-p)\left(n+p-\left|\beta_{1}\right|\right)}\left|f_{n}\right|= \\
=\frac{\left|\beta_{0}\right| k(p+1-\alpha)}{2 p-\left|\beta_{1}\right|}+\frac{\left|\beta_{0}\right|(k-1)(p+2-\alpha)}{2\left(2 p+1-\left|\beta_{1}\right|\right)}\left|f_{p+1}\right|+ \\
+\sum_{n=p+1}^{p+n+1-p)\left(n+p-\left|\beta_{1}\right|\right)}\left|f_{n}\right|-\frac{(p+2-\alpha)(k-1)\left|\beta_{0}\right|}{2\left(2 p+1-\left|\beta_{1}\right|\right)}\left|f_{p+1}\right| \leq \\
\leq \frac{\left|\beta_{0}\right| k(p+1-\alpha)}{2 p-\left|\beta_{1}\right|}+\sum_{n=p+1}^{p+k} \frac{(n+1-\alpha)(k-1)\left|\beta_{0}\right|}{(n-\alpha)(n+1-p)\left(n+p-\left|\beta_{1}\right|\right)}(n-\alpha)\left|f_{n}\right|,
\end{gathered}
$$

whence as above we obtain

$$
\left(1-\frac{(p+2-\alpha)(k-1)\left|\beta_{0}\right|}{2(p+1-\alpha)\left(2 p+1-\left|\beta_{1}\right|\right)}\right) \sum_{n=p+1}^{p+k}(n-\alpha)\left|f_{n}\right| \leq \frac{\left|\beta_{0}\right| k(p+1-\alpha)}{2 p-\left|\beta_{1}\right|} .
$$

If

$$
\frac{(p+2-\alpha)(k-1)\left|\beta_{0}\right|}{2(p+1-\alpha)\left(2 p+1-\left|\beta_{1}\right|\right)}<1
$$


then we get

$$
\sum_{n=p+1}^{p+k}(n-\alpha)\left|f_{n}\right| \leq \frac{\frac{\left|\beta_{0}\right| k(p+1-\alpha)}{2 p-\left|\beta_{1}\right|}}{1-\frac{(p+2-\alpha)(k-1)\left|\beta_{0}\right|}{2(p+1-\alpha)\left(2 p+1-\left|\beta_{1}\right|\right)}}
$$

Similarly,

$$
\begin{aligned}
& \sum_{n=p+1}^{p+k} n(n-\alpha)\left|f_{n}\right| \leq \frac{\left|\beta_{0}\right| k(p+1)(p+1-\alpha)}{2 p-\left|\beta_{1}\right|}+\frac{\left|\beta_{0}\right|(k-1)(p+2)(p+2-\alpha)}{2\left(2 p+1-\left|\beta_{1}\right|\right)}\left|f_{p+1}\right|+ \\
& \quad+\sum_{n=p+3}^{p+k} \frac{n(n-\alpha)(p+k+1-n)\left|\beta_{0}\right|}{(n-p)\left(n+p-1-\left|\beta_{1}\right|\right)}\left|f_{n-1}\right|= \\
& =\frac{\left|\beta_{0}\right| k(p+1)(p+1-\alpha)}{2 p-\left|\beta_{1}\right|}+\frac{\left|\beta_{0}\right|(k-1)(p+2)(p+2-\alpha)}{2\left(2 p+1-\left|\beta_{1}\right|\right)}\left|f_{p+1}\right|+ \\
& +\sum_{n=p+2} \frac{(n+1)(n+1-\alpha)(p+k-n)\left|\beta_{0}\right|}{(n+1-p)\left(n+p-\left|\beta_{1}\right|\right)}\left|f_{n}\right|= \\
& =\frac{\left|\beta_{0}\right| k(p+1)(p+1-\alpha)}{2 p-\left|\beta_{1}\right|}+\frac{\left|\beta_{0}\right|(k-1)(p+2)(p+2-\alpha)}{2\left(2 p+1-\left|\beta_{1}\right|\right)}\left|f_{p+1}\right|+ \\
& +\sum_{n=p+1}^{p+\alpha+1)(n+1-\alpha)(p+k-n)\left|\beta_{0}\right|}\left|f_{n}\right|-\frac{(p+2)(p+2-\alpha)(k-1)\left|\beta_{0}\right|}{2\left(2 p+1-\left|\beta_{1}\right|\right)}\left|f_{p+1}\right| \leq \\
& \leq \frac{\left|\beta_{0}\right| k(p+1)(p+1-\alpha)}{2 p-\left|\beta_{1}\right|}+\sum_{n=p+1}^{p+k} \frac{(n+1)(n+1-\alpha)(k-1)\left|\beta_{0}\right|}{n(n-\alpha)(n+1-p)\left(n+p-\left|\beta_{1}\right|\right)} n(n-\alpha)\left|f_{n}\right|
\end{aligned}
$$

whence

$$
\left(1-\frac{(p+2)(p+2-\alpha)(k-1)\left|\beta_{0}\right|}{2(p+1)(p+1-\alpha)\left(2 p+1-\left|\beta_{1}\right|\right)}\right) \sum_{n=p+1}^{p+k} n(n-\alpha)\left|f_{n}\right| \leq \frac{\left|\beta_{0}\right| k(p+1)(p+1-\alpha)}{2 p-\left|\beta_{1}\right|}
$$

If

$$
\frac{(p+2)(p+2-\alpha)(k-1)\left|\beta_{0}\right|}{2(p+1)(p+1-\alpha)\left(2 p+1-\left|\beta_{1}\right|\right)}<1
$$

then we get

$$
\sum_{n=p+1}^{p+k} n(n-\alpha)\left|f_{n}\right| \leq \frac{\frac{\left|\beta_{0}\right| k(p+1)(p+1-\alpha)}{2 p-\left|\beta_{1}\right|}}{1-\frac{(p+2)(p+2-\alpha)(k-1)\left|\beta_{0}\right|}{2(p+1)(p+1-\alpha)\left(2 p+1-\left|\beta_{1}\right|\right)}} .
$$

Hence we obtain the following statement.

Proposition 2. Let $\alpha \in[0, p), p\left(p+\beta_{1}-1\right)+\gamma_{2}=0, \gamma_{0}=0, k \geq 3, \beta_{0}(p+k)+\gamma_{1}=0$ and $\left|\beta_{1}\right|<2 p$. Then differential equation (3) has a polynomial solution (29), which under the condition

$$
\frac{\left|\beta_{0}\right| k(p+1-\alpha)}{(p-\alpha)\left(2 p-\left|\beta_{1}\right|\right)}+\frac{(p+2-\alpha)(k-1)\left|\beta_{0}\right|}{2(p+1-\alpha)\left(2 p+1-\left|\beta_{1}\right|\right)} \leq 1
$$


is $p$-valent starlike of order $\alpha$ in $\mathbb{D}$ and under the condition

$$
\frac{\left|\beta_{0}\right| k(p+1)(p+1-\alpha)}{\left(2 p-\left|\beta_{1}\right|\right) p(p-\alpha)}+\frac{(p+2)(p+2-\alpha)(k-1)\left|\beta_{0}\right|}{2(p+1)(p+1-\alpha)\left(2 p+1-\left|\beta_{1}\right|\right)} \leq 1
$$

is $p$-valent convex of order $\alpha$ in $\mathbb{D}$.

The conditions (34) and (35) imply (30) and (32), respectively. From (34) and (31) we have (5) and from (35) and (33) we have (6). Therefore, by Lemma 1 Proposition 2 is proved.

\section{REFERENCES}

1. G.M. Goluzin, Geometric theory of functions of a complex variable, M.: Nauka, 1966 (in Russian). Engl. transl.: AMS: Translations of Mathematical monograph, 26 (1969), 676p.

2. W. Kaplan, Close-to-convex schlicht functions, Michigan Math.J., 1 (1952), №2, 169-185.

3. S.M. Shah, Univalence of a function $f$ and its successive derivatives when $f$ satisfies a differential equation II, J. Math. Anal. Appl., 142 (1989), №2, 422-430.

4. Z.M. Sheremeta, The properties of entire solutions of one differential equation, Diff. uravnieniya, 36, (2000), №8, 1045-1050. Engl. transl.: Diff. Equat., 36 (2000), №8, 1155-1161.

5. Z.M. Sheremeta, Close-to-convexity of entire solutions of a differential equation, Mat. method. and fiz.mech. polya, 42 (1999), №3, 31-35. (in Ukrainian)

6. Z.M. Sheremeta, On entire solutions of a differential equation, Mat. Stud., 14 (2000), №1, 54-58.

7. Z.M. Sheremeta, On the close-to-convexity of entire solutions of a differential equation, Visn. Lviv Un-ty. Mech.Math., (2000), №58, 54-56.

8. Z.M. Sheremeta, M.M. Sheremeta, Close-to-convexity for entire solutions of a differential equation, Diff. uravnieniya, 38 (2002), №4, 477-481. Engl. transl.: Diff. Equat., 38 (2002), №4, 496-501.

9. Z.M. Sheremeta, M.M. Sheremeta, Convexity of entire solutions of a differential equation, Mat. method. and fiz.-mech. polya, 47 (2004), №2, 186-191. (in Ukrainian)

10. Ya.S. Mahola, M.M. Sheremeta, Properties of entire solutions of a linear differential equation of $n$-th order with polynomial coefficients of $n$-th degree, Mat. Stud., 30 (2008), №2, 153-162.

11. Ya.S. Mahola, M.M. Sheremeta, Close-to-convexsity an entire solution of a linear differential equation with polynomial coefficients, Visnyk of the Lviv Univ. Series Mech-Math., (2009), №70, 122-127. (in Ukrainian)

12. Ya.S. Mahola, M.M. Sheremeta, On the properties of entire solutions of linear differential equations with polynomial coefficients, Mat. metody and fiz.-mech. polya, 53 (2010), №4, 62-74 (in Ukrainian). Engl. transl.: J. Math. Sci. 181 (2012), №3, 366-382.

13. S. Owa, On certain classes of p-valent functions with negative coefficients, Simon Stevin, 59 (1985), $385-402$.

14. R.M. El-Ashwah, M.K. Aouf, A.O. Moustafa, Starlike and convexity properties for p-valent hypergeometric functions, Acta Math. Univ. Comenianae, 79 (2010), №1, 55-64.

Ivan Franko National University of Lviv, m.m.sheremeta@gmail.com, yurkotrukhan@gmail.com 